\title{
INDEX
}

acquired character, I 34

Aegilops, 157, 169

Aeschna-see dragonfly

Agropyron, 157, 169

allelism, 323, 403

allelomorphs, 285, 399

Allium, 168

amphiploids, 157 sqq.

anastomosis, 210

anthropology, 1 3-32

anthropometry, 14, $20 \mathrm{sqq}$.

antibiotics, 271

antibody, 2, $288 \mathrm{sqq}$.

antigen, 3, 211 , 288 sqq.

Apodemus, $35^{8}$

apomixis, 71, 74

Armeria, 99

Asellus, 168

Aspergillus, 77, 1 35, 280

Asplenium, 280

asynapsis, $74,89 s q q$.

automixis, 74

auxotrophism, 329

bacteria, 27 I

Barber, H. N., 345

barley, 182

Barlow, F., 278

Basques, i6 sqq.

Bateman, A. J., 285

Bateson, I sqq.

Bayes, 2

Beadle, 274

Beale, 272

Berbers, $16 s q q$.

Billingham, R. E., 201

biochemical mutations, 77 sqq.

biochemical processes, 274

biological synthesis, 275

biometrical analysis, 1 90 sqq.

biometrical genetics, 7,387 sqq.

biopsy specimens, 207

biotopes, $34^{\circ}$

blending inheritance, 320

blood groups, 1 3, I I I , 202, 2 1 3, 243, 288

Boole, 2

bolting, 37 sqq.

Brassica, 285, 300

breeding methods, I 7 I sqq.

Bufton, I 35

butterfly-see Maniola

Cain, A. J., 217

Campanula, 371

Capsella, 285, 291, 306

4 II
Cardamine, 286

Caryophyllaceae, 287

Caspersson, T., 274

Catcheside, D. G., 271

Cavalli, L. L., 3 I 1

Celtic syntax, 32

Cepea, 21 7, 233, 333

Ceylon gerbil-see Tatera

Chi square tests, 259

Chlamydomonas, 287

chloromycetin, 312 sqq.

chromosome aberrations induced by X-ray dosage, $127 s q q$.

chromosome analysis, 134

chromosome breakage, 89 sqq.

chromosome mosaics, 157 sqq.

Chrysanthemum, 135, 365-375

Clethrionomys, $35^{8}$

clover, $\mathbf{S}$-alleles in, 301

colchicine treatment, 158,166

conidia, $78-8$ o, 85 , I 35

Cook, Robert C., 132

Copp, L. G. L., 187

correlated response, 329

Crepis, 89, 92, 286, 301

crossing-over, 247

Crowe, L. K., 136

Crucifers, 285

Cryptogams, 290

Cryptostephanus, 144

daffodils, 137

Darlington, 273

Darwin, 132

Dasyurus, 347

Dawson, G. W. P., 243

De Moivre, 2

Diamond, 2

dioecious plants, 74

dioecy, 286, 297

Diplodia, $3^{8} 3$

disease resistance, 383

Dobzhansky, 274

domestic animals, 31

Dowdeswell, W. H., 99

Dowrick, G. J., 365

dragonfly, 167

Drosophila, 8, 73, 92, 134, 28o, 333, 353, 36o, 403

drug-resistance, 3 I I

Dryopteris, 280

earthworms, 55

ecological barriers, 07

effective factors, 195, $34^{2}$ 
412

enzyme systems, 326

Ephrussi, 273

epistatic ratios, 259-26 I

epistasy, 306,308

Escherichia coli, 272, 311

factorial method, 3

Falconer, D. S., 255

fermentation, 263

Festuca-Lolium, 167

Feulgen squashes, 346, 358, 37 I

Fisher, R. A., I

fixatives, 345

flatworms, 74

Ford, E. B., 99

Forsythia, 287

fowl nephritis, 278

freemartin, 201-212

Fritillaria, 144

fungi, $77,86,263,27$ I

Fyfe, J. L., 136

\section{Gaertner, I}

galactose, 263 sqq.

Galton, I

gametophytic control, 285, 292, 305

Gauss, 1, 2

gene-conversion, 266

genetic drift, $108,230,333,340$

Geranium, 279

germination, 377

Gibberella, 377

Gordon, I

Gossypium hybrids, I 68

grafting, 45-52

Grüneberg, Hans, 134

Gymnosperms, 290, 29:

gynodioecy, $282 s q q$.

\section{Haberman, 2}

habitat, 236

hamsters, 358-363

Haskell, Gordon, 377

Heath, A. S., 132

Hemmons, L. M., 135

heterocaryosis, $77-87$

heterosis, $377-383$

heterothally, 287

heterothallism, 135

Hill, 2

Holt, H. A., 213

homografts, 201-21 2

Hormogaster, 55

Huxley, 273

hyacinths, 147

incompatibility, 286-308

Irwin, 274

\section{INDEX}

Jackson, W., 279

Jinks, J. L., 77

Kaplan, W. D., 28 I

kernel weight, 187

Knight, I

Kölreuter, I

Labiatae, 287

Lactobacillus, 312

Lamotte, M., 333

Lampkin, G. H., 201

Landsteiner, 3

language, 25-34

Lavoisier, 3

Lederberg, 272

leeches, 74

Lenin, 277

lethal allele, 295-40 r

lethal mutations, 403

leucocytes, 205, 206

Leucojum, 144

Levine, 3

Lewis, D., $13^{6}$

Lilium, 92

linkage, 7, 121, 194

in the mouse, 134, 255

Linum, 287

Little, 274

Luippold, Henry, 127

Lumbricidae, 55-75

Lysenko, 277

Lythrum, ro, 288

Maccacaro, G. A., 3 I I

Macropus-see Wallabia

Mainland, G. B., 259

maize, $89,94,377$

malt extract, 84

Maltese dilution, 398, 40 r

maltose, 264

Mangelsdorf, 274

Maniola, 99

Manton, I., 28o

Marguerite-see Chrysanthemum

marsupial-see Potorous

Mather, 272

mating behaviour, 239

mating systems, 244,275

mating types, 213

Medawar, P. B., 201

Megascolicidae, 55,68

Melandrium, $36 \mathrm{I}$

melanin, 225

Mendel, I, 132

Mesocricetus, $35^{8}$

metabolic disturbance, 96

Michie, Donald, 134

Michurinist theory, 276

microbes, 271 
microbial genetics, $27 \mathbf{I}$

microcytic anaemia, 255

Mirsky, 274

monoecy, 286

Morton, 276

moth-see Panaxia dominula

Mourant, A. E., 13

mouse, 1 34, 247, 395

mucoid colonies, 315, 329

Mucor, 290

Mucorales, 287

Muldal, Sylfest, 55

Narcissi, $\mathrm{r} 37$ sqq.

Naudin, I

Nelder, J. A., 387

neurological defects, $395,40 \mathrm{I}$

Neurospora, 263, 27I

Nicotiana, 78, 86, 92, 285

nitrogen-mustard, 3 I 2

Oenothera, 286 sqq.

Oligochaeta, 55 sqq.

ontogeny, 167

oogenesis, 58,74

opisthotonus, 395

Orbeli, 277

Origanum, 136

Orthoptera, $35^{2}$

Oster, Irwin I., 403

outbreeding mechanism, 287

Oxalis, 306

Paeonia, 368

Palmer, T. P., I 7 I

Panaxia, 239

Paramecium, 272

Parthenium, 29I

parthenogenesis, $7 \mathbf{I}$

pathogens, 379

Pavlov, 277

Pearson, Karl, 5

penicillin, 31 I, 315, 327

Penicillium, 77-87

Perameles, $35^{\circ}$

Penrose, 274

perithecium analysis, 135

phenyl thiocarbamide, 213

Pheretima, 55

philology, 25

phonetics, 28

photoperiodic reaction, $37,5 \mathrm{I}$

phylogeny, 70

physiological selection, 2 I 7

pigment, 2 I $8,225,260,397,398$

Pilkington, R., 277

Pisum, 81, 91, 92

pleiotropic genes, 401

Plumbaginaceae, 279

Polychaeta, 69 polyploid mammals, 357

polyploidy in Lumbricidae, $7 \mathbf{I}$

Polypodium, 280

Pontecorvo, G., 135, 28o

potato, 37-5?

Potorous, 345

precocity theory, 167

predatory selection, $217,227,228,230$

Primula, 289

probability, I

" pro-gene," $26_{5}$

protandry, 286

protogyny, 286

prototrophs, 3 I $3-330$

Protozoa, $27 \mathrm{I}$

Prunus, 289

pseudo-alleles, 400

pseudo-compatibility, 286, 29I

Pteridophyta, 280

pulmonate Molluscs, 74, 75

Race, R. R., III, 2 I3

raffinose, $265^{-268}$

rainfall, 107

Raphanus, 285

rat kangaroo-see Potorous

" Reeler" in the mouse, 255

Rees, Hubert, 89

Reeve, E. C. R., 280

Robertson, Forbes W., I 34

rogues, $5^{I}$

Ribes nigrum, 166

Roper, J. A., 280

Rosa, 368

rye, $167,37 \mathrm{r}$

Saccharomyces, 263-268

Sachs, Leo, I 57, 357

salivary gland, 403

Sánchez-Monge, I 2 I

Sanger, R., I I I , 2 I 3

saprophyte, 78

Sax, Karl, 127

scent, 240

Schizosaccharomyces, 263

Schizophyllum, 77

Schultz, 274

Scilla, 89-96

Searle, A. G., 395

Secale, $\mathrm{I} 67,37 \mathrm{I}$

segregation, 387

selection coefficients, 230

self-incompatibility, 285-309

serological characters, 271

sex-chromosomes, 352

Sharman, G. B., 345

Sheppard, P. M., 233, 239

Shimotomai, I 35

Shivas, M. G., 280

shrew-see Sorex

skeletal anomaly, I 34 
skin grafting, $20 \mathrm{I}-2 \mathrm{II}$

snail-see Cepea nemoralis

Snell, G. D., 247

sodium azide, 3 I I

soil-types, 107

Solanum, 38, 50

Somme, $34^{1}$

Sonneborn, 272-274

Sorex araneus, 345 sqq.

soya bean, 182

Spector, 278

sporophytic control, 285 sqq.

Stalin, 277

Stanton, W. R., 37

Staphylococcus, 31 I, 327

Staticeae, 279

statistical methods, $1-12$

streptomycin, 311,317

Sturtevant, 274

sucrose, $265-268$

Swann, 409

sweet corn, 377-384

synchorial condition, 202

syndrome, 395

systematics, 68,365

Tapeinanthus, 144

tapetal cells, 168, 293

Tatera indica, $35^{8}$

temperature, 127

in maize germination, 378

tetrad analysis, 272

tetrads, $26_{3} s q q$.

Thompson, J. S., 213

tobacco, 276

Tradescantia, 91, 127-1 30

"training," 312-318, 326

transition matrix, 388,39 r
Trillium, $9 \mathrm{I}$

Trioclopis, 75

Triticum vulgare, 157, 169, I 71-184, 187-199

Tulipa orphanidea, 89

tumour transplants, 247

Turbellaria, 74

twin diagnosis, 20I-2 12

twins, III, I35, 201, 410

velvet technique, 324

Vicia Faba, 136

viruses, 47, 271, 274

Vitamin $\mathbf{B}_{\mathbf{1}}, 3^{1} 3$

vole-see Clethrionomys

Waddington, C. H., 134

Wales, I 3-32

Walker, S., 280

Wallabia, 345,353

Watkin, I. Morgan, I3

Waymouth, 409

Weismann, I

wheat, 173, 187, 197

Williams, H. Ll., 201

Winge, $\boldsymbol{\varphi}, 263$

winter beans-see Vicia Faba

Wright, G. M., 187

Wright, Sewall, 337, 343

Wylie, Ann P.,

yeasts, $263-268,27 \mathrm{I}$

Zea mays-see maize

zygotes, 280, 296 